\title{
Reduced water permeability and altered ultrastructure in thin descending limb of Henle in aquaporin-1 null mice
}

\author{
Chung-Lin Chou, ${ }^{1}$ Mark A. Knepper, ${ }^{1}$ Alfred N. van Hoek, ${ }^{2}$ Dennis Brown, ${ }^{2}$ \\ Baoxue Yang, ${ }^{3}$ Tonghui Ma, ${ }^{3}$ and A.S. Verkman ${ }^{3}$ \\ ${ }^{1}$ Laboratory of Kidney and Electrolyte Metabolism, National Institutes of Health, Bethesda, Maryland 20892, USA \\ ${ }^{2}$ Renal Unit and Program in Membrane Biology, Massachusetts General Hospital East, Boston, Massachusetts 02114, USA \\ ${ }^{3}$ Departments of Medicine and Physiology, Cardiovascular Research Institute, University of California-San Francisco, \\ San Francisco, California 94143-0521, USA \\ Address correspondence to: Alan S. Verkman, 1246 Health Sciences East Tower, Cardiovascular Research Institute, \\ University of California-San Francisco, San Francisco, California 94143-0521, USA. Phone: (415) 476-8530; Fax: (415) 665-3847; \\ E-mail: verkman@itsa.ucsf.edu; http://www.ucsf.edu/verklab
}

Received for publication November 3, 1998, and accepted in revised form January 4, 1999.

\begin{abstract}
It has been controversial whether high water permeability in the thin descending limb of Henle (TDLH) is required for formation of a concentrated urine by the kidney. Freeze-fracture electron microscopy (FFEM) of rat TDLH has shown an exceptionally high density of intramembrane particles (IMPs), which were proposed to consist of tetramers of aquaporin-1 (AQP1) water channels. In this study, transepithelial osmotic water permeability $\left(\mathrm{P}_{\mathrm{f}}\right)$ was measured in isolated perfused segments $(0.5-1 \mathrm{~mm})$ of TDLH in wild-type (+/+), AQP1 heterozygous (+/-), and AQP1 null (-/-) mice. $\mathrm{P}_{\mathrm{f}}$ was measured at $37^{\circ} \mathrm{C}$ using a 100 $\mathrm{mM}$ bath-to-lumen osmotic gradient of raffinose, and fluorescein isothiocyanate (FITC)-dextran as the luminal volume marker. $P_{\mathrm{f}}$ was (in $\left.\mathrm{cm} / \mathrm{s}\right): 0.26 \pm 0.02([+/+] ; \mathrm{SE}, n=9$ tubules), $0.21 \pm 0.01([+/-] ; n=12)$, and $0.031 \pm 0.007([-/-] ; n=6)(P<0.02,[+/+]$ vs. $[+/-] ; P<0.0001,[+/+]$ vs. $[-/-])$. FFEM of kidney medulla showed remarkably fewer IMPs in TDLH from $(-/-)$ vs. $(+/+)$ and $(+/-)$ mice. IMP densities were (in $\mu \mathrm{m}^{-2}$, $\mathrm{SD}, 5-12$ micrographs): 5,880 $\pm 238(+/+) ; 5,780 \pm 450(+/-)$; and $877 \pm 420(-/-)$. IMP size distribution analysis revealed mean IMP diameters of $8.4 \mathrm{~nm}([+/+]$ and $[+/-])$ and $5.2 \mathrm{~nm}([-/-])$. These results demonstrate that AQP1 is the principal water channel in TDLH and support the view that osmotic equilibration along TDLH by water transport plays a key role in the renal countercurrent concentrating mechanism. The similar $\mathrm{P}_{\mathrm{f}}$ and AQP1 expression in TDLH of $(+/+)$ and $(+/-)$ mice was an unexpected finding that probably accounts for the unimpaired urinary concentrating ability in $(+/-)$ mice.
\end{abstract}

J. Clin. Invest. 103:491-496 (1999)

\section{Introduction}

The thin descending limb of Henle (TDLH) in kidney has been proposed to have an important role in the formation of a concentrated urine by the countercurrent multiplication mechanism. Countercurrent multiplication relies on active solute transport out of the lumen in the thick ascending limb of Henle, rapid osmotic equilibration along the lumen of TDLH, and efficient uptake of water by the renal microvasculature $(1,2)$. Measurements from several laboratories indicate that transepithelial osmotic water permeability $\left(\mathrm{P}_{\mathrm{f}}\right)$ in TDLH is exceptionally high. Early split-oil droplet measurements showed rapid osmotically driven water movement into the lumen of TDLH (3), and subsequent in vivo and in vitro microperfusion studies showed very high $\mathrm{P}_{\mathrm{f}}$ values of $>0.2 \mathrm{~cm} / \mathrm{s}$ (reviewed in ref. 4), substantially greater than $\mathrm{P}_{\mathrm{f}}$ in the proximal tubule and the vasopressin-stimulated collecting duct. The transport of water through aqueous pores in TDLH cell membranes was suggested from the findings of a high ratio of osmotic-to-diffusional water permeability and of inhibition of water permeability by the mercurial $p$-chloromercuribenzene sulfonate (5). It was also shown that water permeability in TDLH depends on nephron type (long-loop vs. shortloop nephrons) and position along the tubule axis $(6,7)$.
Water permeability is greatest in the proximal portion of TDLH in long-loop nephrons and becomes low in deep medullary segments.

A possible molecular basis for the high water permeability in TDLH was suggested by freeze-fracture electron microscopy (FFEM) studies showing higher densities of intramembrane particles (IMPs) in plasma membranes of TDLH compared with other nephron segments $(8,9)$. The majority of the IMPs in plasma membranes of rat TDLH were subsequently shown to consist of tetramers of aquaporin-1 (AQP1) water channels (10). AQP1 is a water-selective transporting protein that is expressed widely in fluid-transporting epithelia and endothelia (reviewed in refs. 11, 12). In kidney, AQP1 is expressed at the apical and basolateral plasma membranes of epithelial cells in proximal tubule and TDLH, and in endothelial cells of descending vasa recta (13-16). Consistent with these observations, measurement of AQP1 protein in microdissected tubule segments using a fluorescencebased enzyme-linked immunosorbent assay (ELISA) method showed that TDLH of long-looped nephrons have the highest AQP1 content among nephron segments (17). Recently, transgenic knockout mice deficient in AQP1 were generated by targeted gene disruption and found to be polyuric and unable to concentrate their 
urine in response to water deprivation (18). The inability of vasopressin to increase urine osmolality in the knockout mice was taken as evidence for defective countercurrent multiplication. Analysis of proximal tubule function in AQP1 knockout mice by in vitro microperfusion and in vivo micropuncture indicated fivefold decreased water permeability and $\sim 50 \%$ decreased isosmolar fluid absorption (19). However, the profound urinary concentrating defect in the AQP1 null mice is probably not the consequence of proximal tubule dysfunction, as distal fluid delivery was relatively unaffected because of tubuloglomerular feedback and a compensatory decrease in glomerular filtration rate. It was proposed that defective TDLH and/or descending vasa recta function were primarily responsible for the urinary concentrating defect.

The purpose of this study was to characterize osmotic water permeability and ultrastructure in TDLH of wildtype $(+/+)$, AQP1 heterozygous $(+/-)$, and AQP1 null $(-/-)$ mice. $\mathrm{P}_{\mathrm{f}}$ was measured by in vitro tubule microperfusion, and membrane ultrastructure was studied by FFEM. The principal finding was a profoundly decreased $\mathrm{P}_{\mathrm{f}}$ and IMP particle density in TDLH from the AQP1 null mice. The results have important implications regarding the mechanism of osmotic equilibration along the TDLH and the role of high TDLH water permeability in the urinary concentrating mechanism.

\section{Methods}

Transgenic mice. Transgenic knockout mice deficient in AQP1 protein were generated by targeted gene disruption as described previously (18). When given free access to water and a low-fat diet, the knockout mice appear to be grossly normal except that they are generally $\sim 10 \%$ smaller by weight than litter-matched $(+/+)$ mice. Measurements were done in tissues from littermatched mice (6-8 weeks of age) produced by intercrossing of AQP1 (+/-) mice in a CD1 background. Genotype analysis of tail DNA was done by PCR at 5 days of age. Urine collections during water deprivation and osmolality measurements were done as described previously (18). The mice were deprived of both food and water. Urine collections were done while the mice were being observed in order to transfer urine samples to small closed containers before evaporation occurred. The investigators were blinded to genotype information for tubule water permeability measurements and electron microscopy. The protocols for these studies were approved by the University of California, San Francisco Animal Research Committee.

In vitro TDLH tubule microperfusion. TDLH segments (0.5-1 $\mathrm{mm}$ length) were microdissected from freshly excised kidneys by standard procedures. The isolated tubules were mounted on glass pipettes and perfused in vitro at $37^{\circ} \mathrm{C}$. Transepithelial water flux in response to a $100 \mathrm{mOsm} / \mathrm{kg} \mathrm{H}_{2} \mathrm{O}$ bath-to-lumen osmolality gradient of raffinose was measured to determine the transepithelial osmotic water permeability coefficient $\mathrm{P}_{\mathrm{f}}$ (20). The lumen perfusate consisted of (in $\mathrm{mM}$ ) $\mathrm{NaCl} 120$, $\mathrm{NaHCO}_{3} 25, \mathrm{~K}_{2} \mathrm{HPO}_{4} 2, \mathrm{CaCl}_{2} 2, \mathrm{MgSO}_{4} 1.2$, glucose 5.5 containing $5 \mathrm{mg} / \mathrm{ml}$ FITC-dextran (mol wt 10,000; Molecular Probes, Eugene, Oregon, USA) as a membrane-impermeant volume marker. The fluorescence signal of perfusate and collected samples was measured by a continuous-flow fluorometer as described previously (21).

Immunostaining and immunoblot analysis. For immunostaining, kidneys were removed, sliced, and fixed in $4 \%$ paraformaldehyde for $4 \mathrm{~h}$. Cryostat sections $(4-6 \mu \mathrm{m})$ were cut and stained with a polyclonal anti-AQP1 antibody as described previously (13). Immunoblot analysis was done on kidney homogenates in which cortex and inner medulla were separated. Samples were homogenized in saline containing multiple protease inhibitors, resolved on SDS-PAGE, and electrotransferred and immunoblotted as described previously (18). Quantitative band integration was done by enhanced chemiluminescence and densitometry. Multiple standards were included in all blots to correct for film nonlinearity (see Results).

Freeze-fracture electron microscopy. Kidneys were fixed in $2 \%$ glutaraldehyde for $2 \mathrm{~h}$, washed, and stored in PBS containing $0.01 \% \mathrm{NaN}_{3}$. Small tissue fragments from the base of the papilla were infiltrated with $30 \%$ glycerol in $0.1 \mathrm{M}$ cacodylate buffer ( $\mathrm{pH}$ 7.4) overnight, placed on copper specimen holders, and frozen in $\mathrm{N}_{2}$-cooled Freon-22. Frozen samples were transferred into a freeze-fracture apparatus (Cressington Scientific Instruments, Watford, United Kingdom). Sample temperature was raised to $-120^{\circ} \mathrm{C}$, and membranes were fractured under vacuum $\left(10^{-7}\right.$ torr $)$. Fractured membranes were shadowed at 45 degrees with platinum, followed by a carbon coat perpendicular to the fracture plane. Specimens were digested with sodium hypochlorite solution and then rinsed with distilled water. The replicas were examined on nickel grids in a Philips CM10 electron microscope (Mahwah, New Jersey, USA).

TDLH were identified based on established criteria (22): the presence of numerous short microvilli at the luminal surface of thin interdigitating epithelial cells, numerous basolateral infoldings, tight junctions consisting of a single thick fibril, and the presence of relatively few large areas of plasma membrane produced by the fracture process. IMP densities of protoplasmic $(\mathrm{P})$-face micrographs were quantified by counting particles in $0.1 \times 0.1 \mu \mathrm{m}^{2}$ areas of plasma membrane at $72,000 \times$.

\section{Results}

Figure 1 a shows immunoperoxidase of kidney cortex and medulla from $(+/+),(+/-)$, and (-/-) mice. AQP1 protein was expressed at qualitatively comparable levels in proximal tubule, TDLH, and descending vasa recta in kidneys from $(+/+)$ vs. (+/-) mice. There was no detectable AQP1 expression in the $(-/-)$ mice. Immunoblot analysis showed AQP1 protein expression in kidney from $(+/+)$ and $(+/-)$ mice with no detectable expression in (-/-) mice (Fig. $1 b$, left). AQP1 protein was seen as a narrow band at $\sim 28 \mathrm{kDa}$ corresponding to nonglycosylated AQP1 monomers, and a diffusely migrating band at higher apparent molecular size corresponding to glycosylated AQP1 monomers. Quantitative densitometry of immunoblots was done by comparing integrated band intensities from $(+/-)$ mice to different dilutions of samples from $(+/+)$ mice. As seen qualitatively in Fig. $1 b$, the amount of AQP1 protein in $(+/-)$ was $70 \%-80 \%$ of that in $(+/+)$ mice, a consistent finding in immunoblots from four sets of mice. The averaged quantitative data indicated AQP1 protein expression was reduced in (+/-) vs. $(+/+)$ mice by $28 \pm 9 \%$ (renal cortex) and $22 \pm 14 \%$ (renal medulla) (Fig. $1 b$, right).

Figure 2 shows the time course of urine osmolality in response to water deprivation. Measurements were done on mice of the same age group as used for the water permeability and ultrastructure studies. The (+/+) and (+/-) mice produced a progressively more concentrated urine, with maximum osmolality $>3,000 \mathrm{mOsm}$. The urine concentrating abilities of $(+/+)$ and $(+/-)$ mice did not differ significantly. There was no further increase in urine osmolalities after intraperitoneal injection of the V2-selective vasopressin agonist 1-deamino-8-D-arginine 

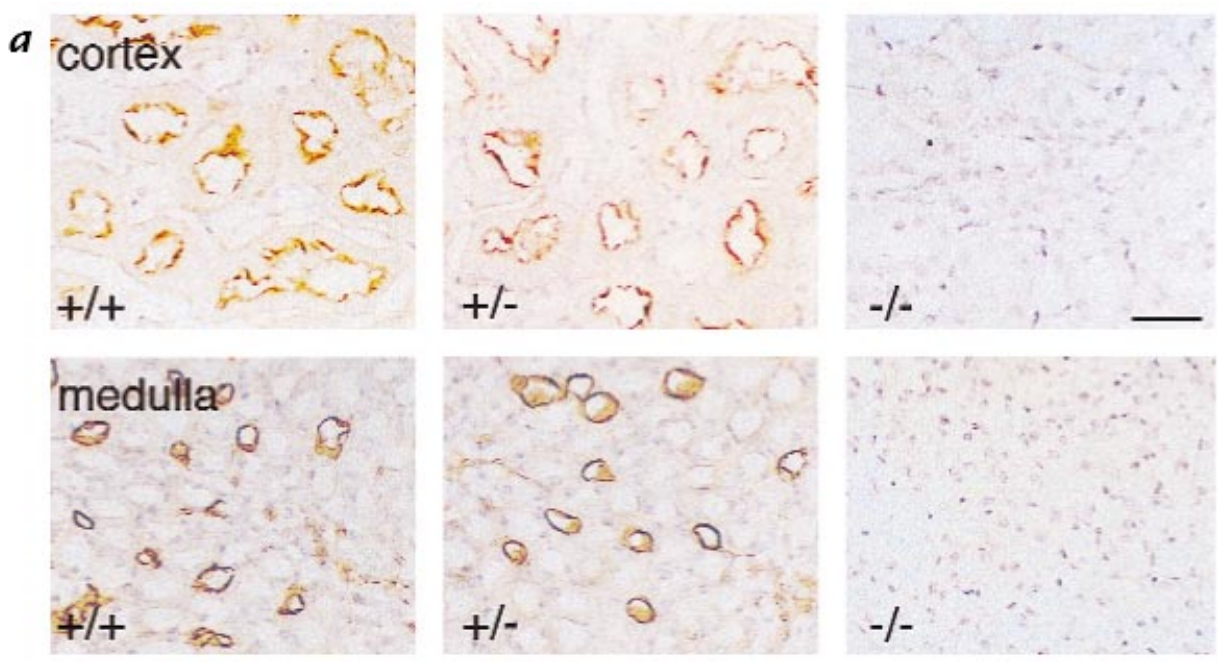

Figure 1

AQP1 expression in kidneys of $(+/+)$, $(+/-)$, and $(-/-)$ mice. (a) Immunoperoxidase localization of AQP1 protein in renal cortex and medulla for mice of indicated genotype. Scale bar, $40 \mu \mathrm{m}$. (b) Left: Representative immunoblot analysis of renal cortex from mice of indicated genotype. A series of dilutions of samples from $(+/+)$ mice are included as indicated. Right: AQP1 protein expression in renal cortex and medulla (SE, $n=4$ mice in each group) determined by quantitative densitometry using standards. $(+/+)$, wild-type mice; $(+/-)$, AQP1 heterozygous mice; $(-/-), A Q P 1$ null mice; $A Q P 1$, aquaporin-1.

$\boldsymbol{b}$

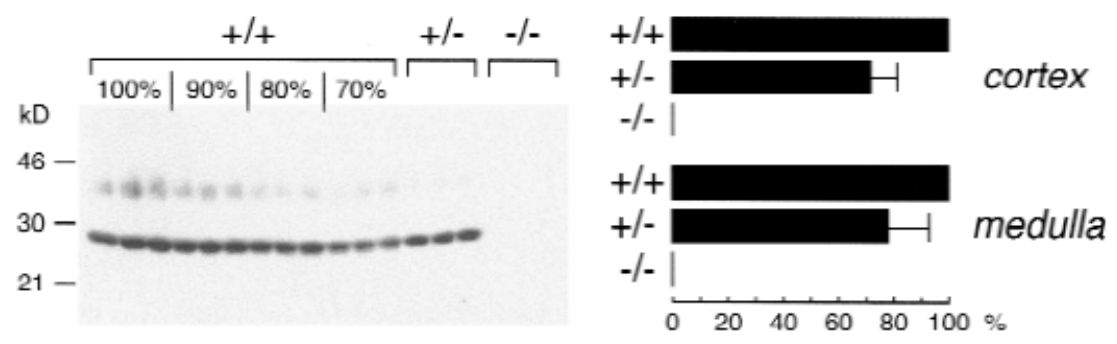

vasopressin. The $(+/+)$ and $(+/-)$ mice remained active and were often hyperactive after a 36-hour water deprivation. In contrast, the (-/-) mice were unable to increase their urine concentration at any time and generally became lethargic after 24 hours. Greater than $80 \%$ of the water deprived (-/-) mice fully recovered when given access to water.

Segments of TDLH from long-loop nephrons were microdissected from the inner medulla near the inner-outer medullary junction and perfused in vitro as described in Methods. Discrimination of inner medullary thin descending limbs was made on the basis of the continuity of thin descending limbs with outer medullary thin descending limbs, whereas ascending limbs connect to thick ascending limbs. Figure 3 shows $\mathrm{P}_{\mathrm{f}}$ values for individual tubules. Averaged $\mathrm{P}_{\mathrm{f}}$ (in $\mathrm{cm} / \mathrm{s}$ ) was $0.26 \pm 0.02(+/+), 0.21 \pm 0.01(+/-)$, and $0.031 \pm 0.007$ $(-/-)$. Statistical analysis (ANOVA) indicated a small but significant difference in $\mathrm{P}_{\mathrm{f}}$ of TDLH of $(+/+)$ vs. $(+/-)$ mice $(P<0.02)$, and a significant 8.5 -fold decrease in $\mathrm{P}_{\mathrm{f}}$ of TDLH of $(+/+)$ vs. $(-/-)$ mice $(P<0.0001)$.

Representative FFEMs of the P-face plasma membrane of TDLH epithelial cells are shown in Fig. 4. As shown previously (22), this segment of the nephron yields mostly cross-fractured epithelial cells, with only small areas of the plasma membrane exposed in the fracture plane. On the right of each micrograph, the small area represents the lumen of the tubule. Adjacent to the lumen, the apical membrane appears more undulating and contains IMPs that represent integral membrane proteins. There was a remarkably different appearance of the TDLH api- cal membrane of (-/-) mice, with a much lower IMP density and different IMP appearance. Figure 5 summarizes the results of IMP counting and size determination. IMP densities were $5,880 \pm 238(+/+) ; 5,780 \pm 450(+/-)$; and $877 \pm 420(-/-)(P<0.001$ for (-/-) vs. others). Size distribution analysis indicated that the predominant IMP size in TDLH membranes of $(+/+)$ and $(+/-)$ mice was 8.4 $\mathrm{nm}$, in agreement with the size of tetrameric AQP1 IMPs reported by Verbavatz et al. (10). Average IMP size in TDLH of $(-/-)$ mice was $5.2 \mathrm{~nm}$.

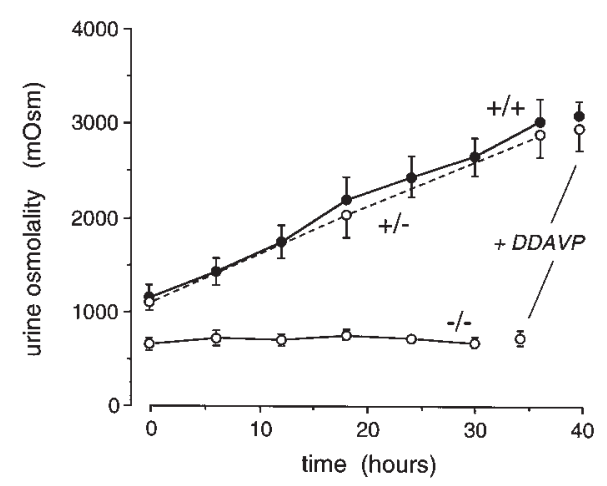

Figure 2

Urinary concentrating ability in (-/-) mice. Mice were deprived of food and water at time 0 . Urine osmolalities were mean \pm SE of measurements for three to six mice in each genotype group. Where indicated, 1-deamino8-D-arginine vasopressin (DDAVP; $0.4 \mu \mathrm{g} / \mathrm{kg}$ ) was given by intraperitoneal injection, and urine osmolality was measured after $1-3 \mathrm{~h}$. 


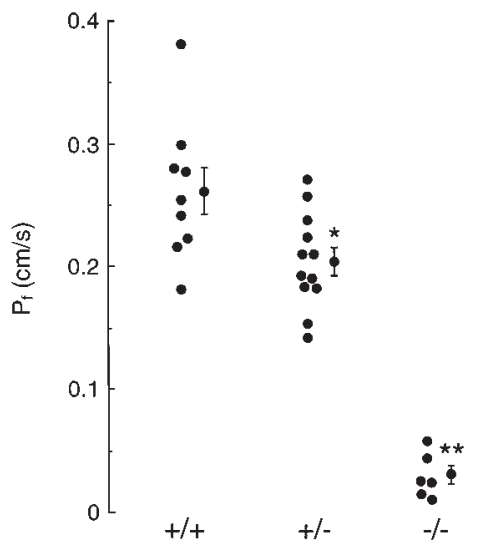

Figure 3

Osmotic water permeability in isolated microperfused TDLH. Transepithelial osmotic water permeability $\left(P_{f}\right)$ was determined at $37^{\circ} \mathrm{C}$ in response to a bath-to-lumen osmotic gradient of $100 \mathrm{mM}$ raffinose as described in Methods. Pf from individual microperfused tubules is shown, along with averaged data (SE). ${ }^{*} P<0.02,{ }^{*} P<0.0001$. TDLH, thin descending limb of Henle.

Figure 6 summarizes averaged $\mathrm{P}_{\mathrm{f}}$ and IMP density for mice of the three genotypes. The $\mathrm{P}_{\mathrm{f}}$ and IMP density of TDLH of (+/-) mice were well above the average of the corresponding values for $(+/+)$ and $(-/-)$ mice, consistent with the expression data in Fig. $1 b$ (see Discussion).

\section{Discussion}

Functional measurements indicated that transepithelial $\mathrm{P}_{\mathrm{f}}$ in TDLH of $(+/+)$ mice was very high $\left(\mathrm{P}_{\mathrm{f}}=0.26 \mathrm{~cm} / \mathrm{s}\right)$

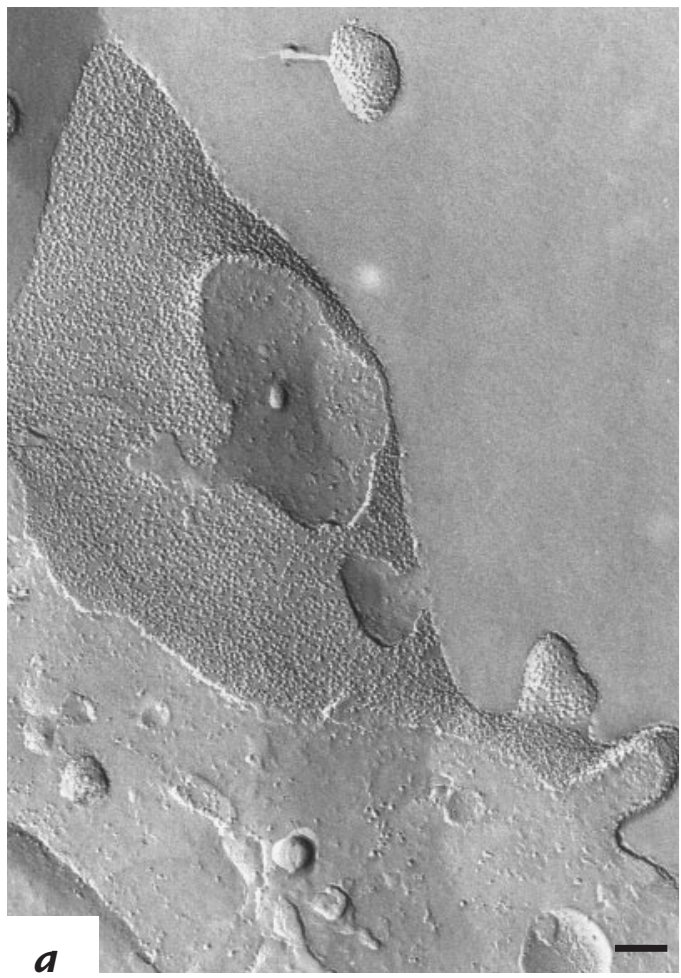

and reduced 8.5 -fold by AQP1 deletion. This finding provides strong evidence that osmotically driven water transport in TDLH is primarily transcellular and mediated by AQP1 water channels in TDLH epithelial cell plasma membranes. The water permeability coefficient after AQP1 deletion was $0.031 \mathrm{~cm} / \mathrm{s}$ at $37^{\circ} \mathrm{C}$. For a smooth membrane, this residual $\mathrm{P}_{\mathrm{f}}$ is relatively high and is consistent with the possibility that TDLH contains small numbers of water channels other than AQP1. To date, only AQP1 has been localized to TDLH. Type I and type III descending limbs in rats contain urea transporters (23), which might transport some water (24), and transcript-encoding water channel AQP6 is expressed strongly in renal medulla (25), but the protein has not yet been localized. A second explanation for the relatively high $\mathrm{P}_{\mathrm{f}}$ in TDLH lacking AQP1 is nonzero paracellular water permeability. A third and more likely explanation is water movement through the lipid portion of the convoluted apical and basolateral membranes. Electron micrographs show some infoldings of apical and basolateral plasma membranes of TDLH epithelial cells (26). Although quantitative measurements have not been reported, the amount of membrane redundancy (folding factor) is much less than that of proximal tubule. If the folding factor for TDLH were 5 , then the corrected $\mathrm{P}_{\mathrm{f}}$ in TDLH of $(-/-)$ mice would be $\sim 0.005 \mathrm{~cm} / \mathrm{s}$, which is in the range expected for water transport through lipid membranes.

The FFEMs indicated a remarkably different appearance of TDLH plasma membranes from kidneys of (-/-) vs. $(+/+)$ mice. The most striking difference was the decrease in IMP density in TDLH of AQP1-deficient mice. This finding supports the conclusion from the quantita-

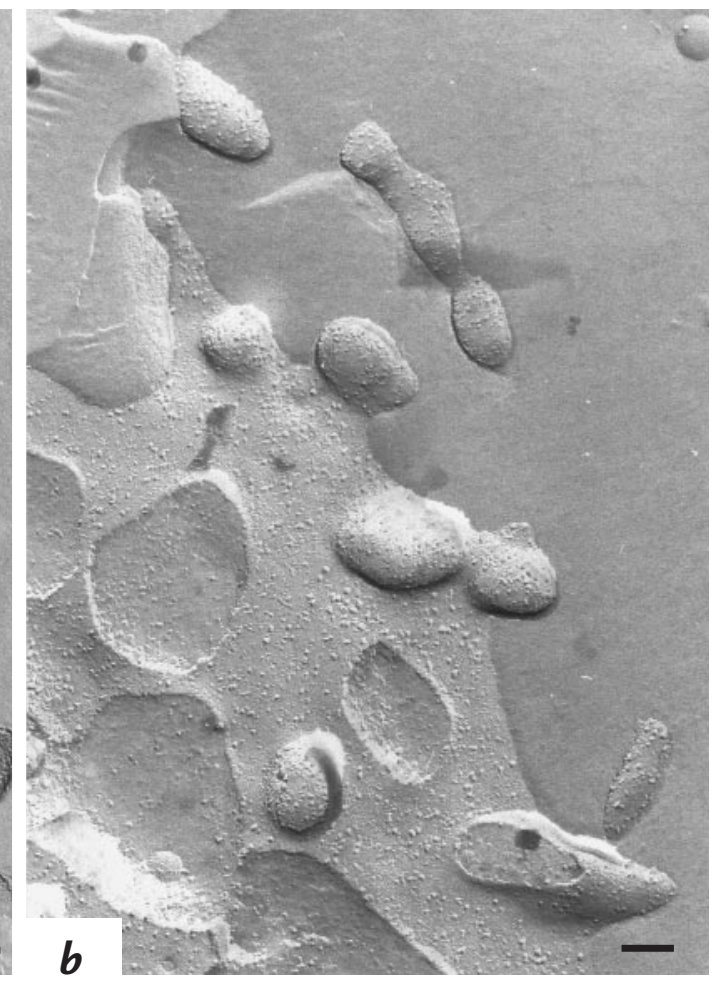

Figure 4

Freeze-fracture electron micrographs of the P-face plasma membranes of TDLH epithelial cells from $(+/+)(\boldsymbol{a})$ and $(-/-)(\boldsymbol{b})$ mice. See the text for explanations. Scale bar, $75 \mathrm{~nm}$. P-face, protoplasmic face. 


\section{Figure 5}

Quantitative analysis of freeze-fracture data. Size distribution of IMPs. Data were fitted to a single Gaussian function as described in Verbavatz et al. (10). Parameters: $(+/+) 1,398 \mathrm{IMPs} \bullet \mu \mathrm{m}^{-2}, 8.4 \mathrm{~nm}$ mean diameter, $2.0 \mathrm{~nm} \mathrm{SD} ;(-/-) 212 \mathrm{IMPs} \bullet \mu \mathrm{m}^{-2}, 5.2 \mathrm{~nm}$ mean diameter, $2.7 \mathrm{~nm}$ SD. IMPs, intramembrane particles. tive analysis of Verbavatz et al. (10) that the majority of IMPs in TDLH plasma membranes are AQP1 tetramers. Comparing IMP densities from $(-/-)$ vs. $(+/+)$ mice, the AQP1 membrane density was $\sim 5,000$ AQP1 tetramers per square micron of plasma membrane. Using a single channel AQP1 water permeability of $10^{-13} \mathrm{~cm}^{3} / \mathrm{s}(27)$, and assuming a folding factor of 5 , the predicted transepithelial TDLH $\mathrm{P}_{\mathrm{f}}$ is $\sim 0.15 \mathrm{~cm} / \mathrm{s}$ at $37^{\circ} \mathrm{C}$. This value is somewhat lower than the measured $\mathrm{P}_{\mathrm{f}}$ of $0.26 \mathrm{~cm} / \mathrm{s}$, possibly because of an underestimate in the folding factor. Rapid water transport is probably the main function of TDLH, as was recently proposed for type I alveolar epithelial cells (28). The remaining low density of IMPs in TDLH plasma membranes of (-/-) mice probably represents the presence of various nonaquaporin solute transporters and other integral membrane proteins.

An unexpected finding was that the water permeability and IMP density of AQP1 (+/-) mice were only mildly reduced compared with those in $(+/+)$ mice. The AQP1 immunoblot analysis indicated that kidneys from $(+/-)$ mice express $22 \%-28 \%$ of the amount of AQP1 protein in kidneys from $(+/+)$ mice. There are several possible explanations for the minimal reduction in AQP1 protein expression in the heterozygous mice. The TDLH might possess a tissue-specific regulatory mechanism at the level of AQP1 transcription or posttranscriptional processing. An intriguing possibility is that the AQP1 density in TDLH is normally so high that membrane packing restrictions might limit the AQP1 plasma membrane density, resulting in a decreased half-life of AQP1 protein in kidneys from $(+/+)$ compared with (-/-) mice. Limited packing could thus produce a nonlinear relationship between

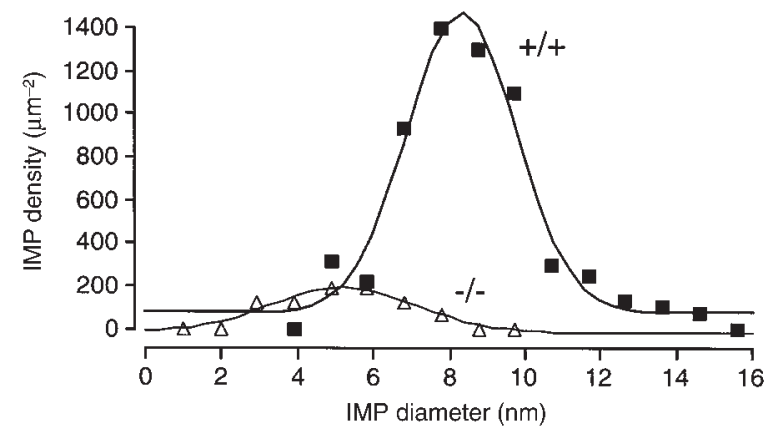

AQP1 membrane density and cellular transcript levels and account for the similar AQP1 densities in TDLH membranes from (+/-) vs. (+/+) mice. Further studies will be needed to distinguish among these possibilities.

There is a longstanding controversy about the relative contributions of water reabsorption vs. solute entry to osmotic equilibration along the TDLH (see ref. 29 for review). Deletion of AQP1 in the null mice produces a profound defect in urinary concentrating ability and decrease in TDLH water permeability, suggesting that high water permeability in the TDLH is necessary for urinary concentration. Although the $\mathrm{NaCl}$ and urea permeabilities of the TDLH have not been measured, they are unlikely to be greatly affected by deletion of AQP1 because AQP1 is not permeable to these solutes $(27,30)$. From these considerations, we conclude that osmotic water transport out of the lumen of TDLH is important for the countercurrent multiplication mechanism and that solute entry is in itself not sufficient to permit the formation of a maximally concentrated urine. Modeling of the concentrating mechanism in the (-/-) mice should be helpful in resolving the quantitative contributions of TDLH water reabsorption vs. solute entry.

The results here do not provide quantitative information about the relative roles of high water permeability in TDLH vs. descending vasa recta for formation of a concentrated urine. Preliminary measurements indicate significantly reduced $\mathrm{P}_{\mathrm{f}}$ in perfused outer medullary descending vasa recta from AQP1 (-/-) mice compared with (+/+) mice (Pallone, T.L., et al., unpublished results). Mathematical modeling might be helpful in quantifying the importance of AQP1 in TDLH vs. vasa recta, as

\section{Figure 6}

Summary of average TDLH $P_{f} v s$. IMP density for mice of indicated genotype. Dashed line intersecting $x$ and $y$ axes shows average of $\mathrm{P}_{\mathrm{f}}$ and IMP density values for $(+/+)$ and $(-/-)$ mice.

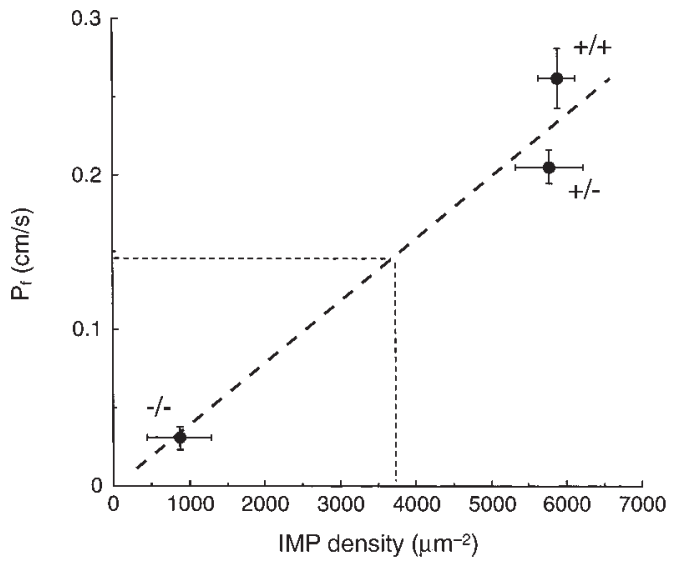


might the tissue-specific deletion of AQP1 in TDLH or renal microvessels. In this regard, recent adenoviral gene delivery experiments (31) are consistent with the conclusion that high TDLH water permeability is an important component of the urinary concentrating mechanism. A few days after intravenous delivery of adenovirus encoding AQP1 to (-/-) mice, AQP1 protein was strongly expressed in hepatic sinusoids and in kidney proximal tubule epithelia and microvascular endothelia. There was no detectable AQP1 protein expression in TDLH, collecting duct, or glomerulus. The adenovirus-treated mice showed partial correction of the urinary concentrating defect, with urine osmolalities increasing from 600-700 mOsm before water deprivation to 1,200-1,400 mOsm after water deprivation, well below that of 2,800-3,200 mOsm seen in (+/+) mice. Because AQP1 protein was expressed strongly in proximal tubule and vasa recta but not in TDLH, these results support the view that high TDLH water permeability is necessary for the formation of a maximally concentrated urine.

\section{Acknowledgments}

The authors thank Jian Yang for animal breeding and genotype analysis, and Maurice B. Burg and Kenneth Spring for helpful discussions and critique. This work was supported by grants DK35124, HL-59198, HL-60288, and DK-43840 (to A.S. Verkman), DK-38452 (to D. Brown), and DK-55864 (to A.N. van Hoek) from the National Institutes of Health, by Research Development Program grant R613 from the National Cystic Fibrosis Foundation (to A.S. Verkman), and by intramural funds from the National Heart, Lung, and Blood Institute (to M.A. Knepper).

1. Moore, L.C., and Marsh, D.J. 1980. How descending limb of Henle's loop permeability affects hypertonic urine formation. Am. J. Physiol. 239:F57-F71

2. Stephenson, J.L., Jen, J.F., Wang, H., and Tewarson, R.P. 1995. Convective uphill transport of $\mathrm{NaCl}$ from ascending thin limb of loop of Henle. Am. J. Physiol. 268:F680-F692.

3. Gottschalk, C.W. 1964. Osmotic concentration and dilution of the urine. Am. J. Med. 36:670-684.

4. Imai, M., Taniguchi, J., and Tabei, K. 1987. Function of thin loops of Henle. Kidney Int. 31:565-579.

5. Miwa, T., and Imai, M. 1983. Flow-dependent water permeability of the rabbit descending limb of Henle's loop. Am. J. Physiol. 245:F743-F754.

6. Imai, M., and Yoshitomi, K. 1990. Heterogeneity of the descending thin limb of Henle's loop. Kidney Int. 38:687-694.

7. Chou, C.L., and Knepper, M.A. 1992. In vitro perfusion of chinchilla thin limb segments: segmentation and osmotic water permeability. Am.J. Physiol. 263:F417-F426.

8. Humbert, F., Pricam, C., Perrelet, A., and Orci, L. 1975. Freeze-fracture differences between plasma membranes of descending and ascending branches of the rat Henle's thin loop. Lab. Invest. 33:407-428.

9. Schiller, A., Taugner, R., and Kriz, W. 1980. The thin limbs of Henle's loop in the rabbit. Cell Tissue Res. 207:249-265.
10. Verbavatz, J.M., et al. 1993. Tetrameric assembly of CHIP28 water channels in liposomes and cell membranes. A freeze-fracture study. J. Cell Biol. 123:605-618.

11. Verkman, A.S., et al. 1996. Water transport across mammalian cell membranes. Am. J. Physiol. 270:C12-C30.

12. Agre, P., et al. 1993. Aquaporin CHIP-the archetypal molecular water channel. Am. J. Physiol. 265:F463-F476.

13. Sabolic, I., et al. 1992. Localization of the CHIP28 water channel in rat kidney. Am. J. Physiol. 263:C1225-C1233.

14. Zhang, R., Skach, W., Hasegawa, H., van Hoek, A.N., and Verkman, A.S. 1993. Cloning, functional analysis and cell localization of a kidney proximal tubule water transporter homologous to CHIP28. J. Cell Biol. 120:359-369.

15. Nielsen, S., Smith, B.L., Christensen, E.I., Knepper, M.A., and Agre, P. 1993. CHIP28 water channels are localized in constitutively water-permeable segments of the nephron. J. Cell Biol. 120:371-383.

16. Nielsen, S., et al. 1995. Aquaporin-1 water channels in short and long loop descending thin limbs and in descending vasa recta in rat kidney. Am. J. Physiol. 268:F1023-F1037.

17. Maeda, Y., Smith, B.L., Agre, P., and Knepper, M.A. 1995. Quantification of aquaporin-CHIP water channel protein in mirodissected renal tubules by fluorescence-based ELISA. J. Clin. Invest. 95:422-428.

18. Ma, T., et al. 1998. Severely impaired urinary concentrating ability in transgenic mice lacking aquaporin-1 water channels. J. Biol. Chem. 273:4296-4299.

19. Schnermann, J., et al. 1998. Defective proximal tubular fluid reabsorption in transgenic aquaporin-1 null mice. Proc. Natl. Acad. Sci. USA. 95:9660-9664.

20. Chou, C.L., Ma, T., Yang, B., Knepper, M.A., and Verkman, A.S. 1998. Four-fold reduction in water permeability in inner medullary collecting duct of aquaporin-4 knockout mice. Am. J. Physiol. 274:C549-C554.

21. Wall, S.M., Han, J.S., Chou, C.L., and Knepper, M.A. 1992. Kinetics of urea and water permeability activation by vasopressin in rat terminal IMCD. Am. J. Physiol. 262:F980-F998.

22. Orci, L., Humbert, F., Brown, D., and Perrelet, A. 1981. Membrane ultrastructure in urinary tubules. Int. Rev. Cytol. 73:183-242.

23. Shayakul, C., Knepper, M.A., Smith, C.P., DiGiovanni, S.R., and Hediger, M.A. 1997. Segmental localization of urea transporter mRNAs in rat kidney. Am. J. Physiol. 272:F654-F660.

24. Yang, B., and Verkman, A.S. 1998. Urea transporter UT3 functions as an efficient water channel: direct evidence for a common water/urea pathway. J. Biol. Chem. 273:9369-9372.

25. Ma, T., Yang, B., Kuo, W.L., and Verkman, A.S. 1996. cDNA cloning and gene structure of a new water channel expressed exclusively in human kidney: evidence for a gene cluster of aquaporins at chromosome locus 12q13. Genomics. 35:543-550.

26. Schwartz, M.M., and Venkatachalam, M.A. 1974. Structural differences in thin limbs of Henle: physiological implications. Kidney Int. 6:193-208.

27. Van Hoek, A.N., and Verkman, A.S. 1992. Functional reconstitution of the isolated erythrocyte water channel CHIP28. J. Biol. Chem. 267:18267-18269.

28. Dobbs, L., et al. 1998. Highly water-permeable type I alveolar epithelial cells confer high water permeability between the airspace and vasculature in rat lung. Proc. Natl. Acad. Sci. USA. 95:2991-2996.

29. Roy, D.R., Layton, H.E., and Jamison, R.L. 1992. Countercurrent mechanism and its regulation. In The kidney: physiology and pathophysiology. D.W. Seldin and G. Giebisch, editors. Raven Press. New York, NY. 1649-1692.

30. Yang, B., and Verkman, A.S. 1997. Water and glycerol permeability of aquaporins 1-5 and MIP determined quantitatively by expression of epitope-tagged constructs in Xenopus oocytes. J. Biol. Chem. 272:16140-16146.

31. Yang, B., Ma, T., Dong, J.Y., and Verkman, A.S. 1998. Partial correction of the urinary concentrating defect in AQP null mice by adenovirusmediated AQP1 gene delivery. J. Am. Soc. Nephrol. 9:29a. (Abstr.) 